\title{
Solid Neoplasm
}

National Cancer Institute

\section{Source}

National Cancer Institute. Solid Neoplasm. NCI Thesaurus. Code C9292.

A benign or malignant neoplasm arising from tissues that do not include fluid areas.

Representative examples include epithelial neoplasms (e.g. lung carcinoma, prostate carcinoma, breast carcinoma, colon carcinoma), and neoplasms arising from the soft tissues and bones (e.g. leiomyosarcoma, liposarcoma, chondrosarcoma, osteosarcoma). Neoplasms originating from the blood or bone marrow (leukemias and myeloproliferative disorders) are not considered solid tumors. 